\title{
JACOBI EVOLUTION OF STRUCTURE FUNCTIONS: CONVERGENCE AND STABILITY
}

\author{
G.Shaw \\ Theoretical Physics Group, Department of Physics and Astronomy, \\ The University of Manchester, M13 9PL, UK
}

(January 7, 1999)

\begin{abstract}
The Jacobi evolution method has been widely used in the QCD analysis of structure function data. However a recent paper claims that there are serious problems with its convergence and stability. Here we briefly review the evidence for the adequate convergence of the method; and show that there are errors in the above paper which undermine its conclusions.
\end{abstract}

\section{INTRODUCTION}

In analysing data on nucleon structure functions, it is convenient to have as fast and simple a method of implementing their QCD evolution as possible. For this reason, the Jacobi evolution method [1 3] has been adopted in many recent analyses円, including applications to polarized [4] and non-forward or off-diagonal [5] parton distributions. However, Ghosh and Raha [6] have made the startling claim that it is unstable due to convergence problems. In this paper, we discuss the convergence properties of the method in general, and the problem raised by Ghosh and Raha in particular.

We start by very briefly summarizing the method [1-3]. The basic idea is to expand a given structure function $\Delta\left(x, Q^{2}\right)$ at fixed $Q^{2}$ in the form

$$
\Delta\left(x, Q^{2}\right)=x^{\beta}(1-x)^{\alpha} \sum_{k=0}^{\infty} a_{k}\left(Q^{2}\right) \Theta_{k}^{\alpha \beta}(x)
$$

where $\alpha, \beta>-1$ are real numbers and $\Theta_{k}^{\alpha \beta}(x)$ are a complete set of Jacobi polynomials of order $k=0,1,2, \ldots$. They are defined to satisfy[

$$
\int_{0}^{1} d x x^{\beta}(1-x)^{\alpha} \Theta_{k}^{\alpha \beta}(x) \Theta_{l}^{\alpha \beta}(x)=\delta_{k l}
$$

\footnotetext{
${ }^{1}$ A SPIRES search reveals more than 30 citations since 1995.

${ }^{2}$ We follow the standard notation and conventions of [2], where further mathematical details may be found.
} 
so that the "Jacobi moments"

$$
a_{k}\left(Q^{2}\right)=\int_{0}^{1} d x \Delta\left(x, Q^{2}\right) \Theta_{k}^{\alpha \beta}(x) .
$$

In practice, the truncated expansion

$$
\Delta\left(x, Q^{2}\right)=\Delta_{N}\left(x, Q^{2}\right) \equiv x^{\beta}(1-x)^{\alpha} \sum_{k=0}^{N} a_{k}\left(Q^{2}\right) \Theta_{k}^{\alpha \beta}(x)
$$

is used, where $N$ is hopefully large enough for this to be a good approximation. The structure function can then be evolved in $Q^{2}$ by using the appropriate QCD evolution equations for the "Jacobi moments." For non-singlet structure functions these are of the form

$$
a_{k}\left(Q^{2}\right)=\sum_{j=0}^{k} E_{j k}^{\alpha, \beta}\left(Q^{2}, Q_{0}^{2}, \Lambda\right) a_{j}\left(Q_{0}^{2}\right)
$$

where the $E_{j k}^{\alpha, \beta}\left(Q^{2}, Q_{0}^{2}, \Lambda\right)$ are known coefficients and $Q_{0}^{2}$ is some conveniently chosen reference $Q^{2}$. Alternatively and equivalently, the Jacobi moments $a_{k}\left(Q^{2}\right)$ can be expressed in terms of the more familiar Cornwall-Norton moments at the reference $Q_{0}^{2}$. For singlet structure functions, the evolution equations are modified by the addition of appropriate inhomogeneous terms dependent on the moments of the gluon distributions at the reference $Q_{0}^{2}$.

\section{CONVERGENCE OF THE JACOBI EXPANSION}

The conditions for uniform convergence [3],8] of the expansion (11) in the range $0<x<1$ are the existence of the integrals

$$
\int_{0}^{1} d x x^{\beta}(1-x)^{\alpha} \Delta\left(x, Q^{2}\right) \quad \int_{0}^{1} d x x^{\beta}(1-x)^{\alpha}\left[\frac{\Delta\left(x, Q^{2}\right)-\Delta\left(z, Q^{2}\right)}{x-z}\right]^{2}
$$

for $0<z<1$. For non-singlet structure functions, assumed to be analytic and bounded, these are satisfied for the whole range of values $\alpha, \beta>-1$ for which Jacobi polynomials exist. For singlet structure functions, assumed to behave like $x^{-\gamma}$ as $x \rightarrow 0$ with $\gamma \geq 0$, they are satisfied for $\alpha>-1, \beta>-1+2 \gamma$. The key question is not whether a convergent series exists, but whether it converges sufficiently rapidly for the truncated expansion (4) to be a good approximation for suitably chosen values of $\alpha$ and $\beta$. These values can reflect the observed behaviour at small and large $x$, or simply be varied in order to improve the convergence.

The accuracy of the truncated expansion (4) can be checked in several different ways. By construction (4.5) automatically guarantees the correct evolution of the first $N$ CornwallNorton moments, but other QCD evolution properties will only be correctedly reproduced if the series (4) has converged. For example, integrals of the form

$$
\int_{0}^{1} d x \Delta\left(x, Q^{2}\right) / x
$$


are sensitive to the low- $x$ region, and correspond to the well-known Gross-Llewellyn-Smith and Gottfried sum rule integrals for $\Delta=x F_{3}$ and $\Delta=F_{2}^{e p}-F_{2}^{e n}$ respectively. These are independent of $Q^{2}$ in LO QCD, but this is only guaranteed by (4) if the series has converged. Barker, Langensiepen and Shaw [2] checked this property in their LO fits and found that with $N=10$ it was satisfied over the range $2 \leq Q^{2} \leq 200 \mathrm{GeV}^{2}$ to within an accuracy of $10^{-3}$. They also checked that the QCD evolution of the higher Cornwall-Norton moments $M_{i}\left(Q^{2}\right)$ for $i=15,20$ was accurately reproduced [3 and concluded that truncating at $N=10$ was sufficient for all practical purposes over the kinematic range considered.

Other methods are to check the variation of the predictions of (田, 5) with increasing $N$; and to compare them with the results of "exact" numerical evolution of the DGLAP equations for the same input. Both methods were exploited for non-singlet strucure functions by Chyla and Rames [7], who compared the results of "exact" QCD evolution with the results obtained by trucating the Jacobi series at $N$ values varying up to 20. Specifically, they assumed the input form

$$
\Delta\left(x, Q^{2}\right)=4.05 x^{0.404}(1-x)^{3.73} \quad\left(Q_{0}^{2}=90\right)
$$

and considered its evolution over the $Q^{2}$ range $1 \leq Q^{2} \leq 1000 \mathrm{GeV}^{2}$. They found a rapidly convergent oscillatory approach to the "exact solution" using the same values $\alpha=3, \beta=1 / 2$ as [2]; and that small variations in these values had little effect on the convergence. For example, detailed results are presented for the reasonable choice $\Lambda_{L O}=0.1$, retaining terms up to $N=9$. Even at $x=0.7$ where the structure function is very small, it is reproduced with an accuracy of $\leq 1 \%$, corresponding to an absolute error of $\leq 0.0005$ This is very small on the scale of variation of the structure function itself, which has a maximum value of order unity.

Chyla and Rames [7] also made similar studies for the other evolution methods which had been proposed and concluded that the Jacobi method was "by far the simplest, fastest and simultaneously very accurate way of analysing the $Q^{2}$ evolution of structure functions."

Finally, we note that for singlet structure functions, Krivokhizhin et al. [3] have presented results for the errors obtained in reproducing a "typical structure function"

$$
\Delta(x)=2.67 x^{0.25}(1-x)^{3}+0.48(1-x)^{8}
$$

at fixed $Q^{2}$ using the truncated expansion (4) for $N$ values up to 17 . For the most favourable values $\alpha=3, \beta=-0.85$ they found an average error $<10^{-4}$ for $N=7$; and for $N=8$, the error at any $x$ was always less than $10^{-4}$. For other $\alpha, \beta$ values the convergence is less rapid but still more than adequate for practical purposes.

\section{A POSSIBLE PROBLEM?}

Very recently, Ghosh and Raha [6] have again investigated the convergence of the Jacobi method by assuming specific analytic forms for non-singlet structure functions at the

\footnotetext{
${ }^{3}$ In LO, the logarithm of the Cornwall-Norton moments depends linearly on $t \ln \left[\ln Q^{2} / \Lambda^{2}\right]$. This linear dependence was reproduced with a fitted slope within one per cent of the QCD prediction.
} 
reference $Q_{0}^{2}$. These are approximated by the expansion (代) using (3) to evaluate the Jacobi moments at $Q_{0}^{2}$ and (5) to evolve them to other $Q^{2}$.

The basic problem is already manifest at the reference $Q^{2}=Q_{0}^{2}$. Plots are presented showing that as $N$ is increased from zero, their computed value of the truncated series $\Delta_{N}\left(x, Q_{0}^{2}\right)$ initially oscillates and then becomes approximately stable and equal to the exact $\Delta\left(x, Q_{0}^{2}\right)$ for a wide range of $N$ values. This is similar to the behaviour found by other authors. However when $N$ increases further, beyond a value which is typically of order 20 25 , the results become wildly unstable, presumably reflecting large contributions from high order polynomials in the Jacobi series (1). They therefore conclude that "the convergence breaks down completely for large $N$."

This reult is surprising, since the input structure functions are smoothly varying and the series must eventually converge uniformly, as noted above. However the precise evaluation of both Jacobi polynomials and of the integrals (3) becomes non-trivial as the order increases; and no tests are reported with a view to verifying the accuracy of the methods used for the highest orders considered. The question therefore arises whether the reported problem is really endemic to the Jacobi method; or whether it could arise from problems with the procedures used, which are not specified in any detail. Fortunately, this can be easily settled using the first set of input forms considered, which are of the generic typef

$$
\Delta\left(x, Q_{0}^{2}\right)=x^{3 / 2}\left(1-x^{2}\right)^{3} \sum_{k=0}^{p} b_{k}\left(1-x^{2}\right)^{k} .
$$

This can obviously be rewritten in the form

$$
\Delta\left(x, Q_{0}^{2}\right)=x^{1 / 2}(1-x)^{3} \sum_{j=1}^{2 p+4} c_{j} x^{j} .=x^{1 / 2}(1-x)^{3} \sum_{j=1}^{2 p+4} a_{j}\left(Q_{0}^{2}\right) \Theta_{j}^{3,1 / 2}(x),
$$

where we have assumed the usual values $\alpha=3 \beta=1 / 2$ for the Jacobi indices. In other words, $a_{k}\left(Q_{0}^{2}\right)=0$ for $k>N_{\max }=2 p+4$ and the Jacobi expansion, implemented in the most straightforward way, terminates exactly after a small number of terms. The problematic contributions found by Ghosh and Sitar at large $N$ are completely excluded in this case. They are not endemic in the Jacobi method, and must arise from some problem with their procedure, numerical or otherwise.

\footnotetext{
${ }^{4}$ Cf. eqns.(11) of [6] for $\Delta=x u_{v}(x), x d_{v}(x)$

${ }^{5}$ Another problem raised in their paper is that even if one restricts oneself to $N$ values in the range where the results are stable, their results are strongly dependent on whether they start their evolution from $Q^{2}=3.5$ or $Q^{2}=5 \mathrm{GeV}^{2}$. This stems from two trivial errors in the equations used to describe the input at $Q^{2}=3.5$. For full details see the earlier and longer version of this paper, which is still accessible on lanl hep-ph/9901253 $\mathrm{v} 1$.
} 


\section{SUMMARY}

Previous work has established that the Jacobi expansion coverges very rapidly for reasonably chosen parameters $\alpha, \beta$. In the light of the previous subsection, we do not believe that Ghosh and Raha have provided any reason to modify this conclusion. 


\section{REFERENCES}

[1] N. Sourlas and G. Parisi, Nucl. Phys. B131 (1979) 421.

[2] I.S. Barker, C.S. Langensiepen and G. Shaw, Nucl. Phys. B186 (1981) 61

[3] V.G. Krivokhizhin et al., Z. Phys. C36 (1987) 51.

[4] E. Leader, A.V.Sidorov and D.B. Stameneov, Phys. Rev. D58 (1998) 114028; C. Bourrely et al. Prog. Theor. Phys. 99 (1998) 1017.

[5] A.V. Belitsky et al hep-ph/9810275 October, 1998.

[6] S.K. Ghosh and S. Raha, lanl hep-ph/9810368 October, 1998.

[7] J. Chyla and J. Rames, Z. Phys. C31 (1986) 151.

[8] G. Szego, Orthogonal Polynomials, American Mathematical Society Colloquium Publications, vol. 23 (New York, 1959). 